\title{
A possibilistic graphical model for handling decision problems under uncertainty
}

\author{
Benferhat Salem $^{1}$ Khellaf Faiza ${ }^{2}$ Zeddigha Ismahane $^{1,2}$ \\ ${ }^{1}$ CRIL, Université d'Artois. Rue Jean Souvraz, sp 18, F62307 Lens, France. \\ ${ }^{2}$ RIIMA, Université des sciences et de la technologie Houari Boumediene, 16111 Bab Ezzouar Algiers, Algeria.
}

\section{Abstract}

Possibilistic networks are important and efficient tools for reasoning under uncertainty. This paper proposes a new graphical model for decision making under uncertainty based on possibilistic networks. In possibilistic decision problems under uncertainty, available knowledge is expressed by means of possibility distribution and preferences are encoded by means another possibility distribution representing the qualitative utility. The first part of the paper proposes a new graphical way to represent such problem, where agent's knowledge and preferences are encoded separately by two distinct possibilistic networks. The first one encodes agent's beliefs and the second one represents the qualitative utility. The second part of the paper proposes a new algorithm for computing optimistic optimal decisions based on merging these two possibilistic networks. In fact, the qualitative possibilistic decision is viewed as a data fusion problem of these two particular possibilistic networks. We show that the computation of optimal decisions comes down to compute a normalization degree of the junction tree associated with the graph representing the fusion of agent's beliefs and preferences.

Keywords: Possibilistic decision theory, min-based possibilistic networks, junction trees.

\section{Introduction}

Decision making under uncertainty plays an important role in Artificial Intelligence (AI) applications. Several decision making tools have been developed to assist decision makers in their tasks: simulation techniques, dynamic programming, logical decision models and graphical decision models.

This paper focuses on graphical decision models which provide efficient decision tools by allowing a compact representation of decision problems under uncertainty [1]. Most of decision graphical models are based on Influence Diagrams (ID) [2] for representing decision maker's beliefs and preferences on sequences of decisions to be made under uncertainty. The evaluation of influence diagrams ensures optimal decisions while maximizing the decision maker's expected utilities. In order to generate optimal decisions, there are two evaluation methods: direct methods [3] that need heavy computations and indirect methods [4] that require a graphical transformation of initial graph into a secondary structure which will be used in different computations. Besides, in many applications, it is easier to express uncertainty in a qualitative way by ranking different states of the world. Similarly, it is more easier to provide a preference relation between different consequences. In these situations, possibility theory [5] is an appropriate framework for representing uncertain knowledge and preferences. Indeed, a qualitative possibilistic decision model [6] allows a gradual expression of both agent's preferences and knowledge.

Few works exist on decision making using possibilistic networks. Garcia and Sabbadin [7] have proposed a possibilistic counterpart of standard influence diagrams. Possibilistic Influence Diagrams (PID) offer a compact representation of problems dealing with decision making under uncertainty. The graphical part of possibilistic influence diagrams is exactly the same as the one of standard influence diagrams. Uncertainty is expressed by possibility degrees and preferences are considered as satisfaction degrees. Unlike probabilistic decision theory which is based on one expected utility criteria to evaluate optimal decisions, a qualitative possibilistic decision theory offers several qualitative utility criteria for decision approaches under uncertainty. Among these criteria, one can mention the pessimistic utility and the optimistic one proposed in [6], the binary utility proposed by $\mathrm{Gi}$ ang and Shenoy [8], etc. In order to evaluate such possibilistic influence diagrams, two ways to accomplish this task, have been proposed in literature: (1) an indirect methods which transform a possibilistic influence diagram either into a decision tree [7] or into a qualitative possibilistic network [9] and (2) a direct methods [7] that use initial structures but require heavy computations in order to update possibility distribution tables.

Our aim in this paper is to propose a new graphical model for representing decision making under uncertainty based on the use of possibilistic networks. When agent's knowledge and preferences are expressed in a qualitative way, we suggest to represent them by two distinct qualitative possibilistic networks. The first one encodes a joint possibility distribution representing available knowledge and 
the second one encodes the qualitative utility.

In a second phase, using our new model for representing qualitative possibilistic decision problems, we present an efficient and unified way of computing optimal optimistic decisions using inference process based on the junction tree associated with the fusion of agent's beliefs and preferences networks. We show that computing optimal decisions comes down to compute a normalization degree of this junction tree. Qualitative possibilistic decision will then be viewed as a data fusion of these two particular possibility distributions (or two qualitative possibilistic networks).

The rest of this paper is organized as follows: next section briefly recalls basic concepts of possibility theory and min-based possibilistic networks. In the same section, we give main results on the data fusion problem of min-based possibilistic networks. Section 3 describes our new model for encoding decision problems based on possibilistic networks. Section 4 describes how propagation process can be efficiently used for computing optimal optimistic decisions. Section 5 concludes the paper.

\section{Backgrounds}

\subsection{Basic concepts of possibility theory}

This section gives a brief refresher on possibility theory [5] which is issued from fuzzy sets theory [11]. Let $\mathcal{V}=\left\{X_{1}, \ldots, X_{N}\right\}$ be a set of variables. We denote by $\mathbb{D}_{X_{i}}=\left\{x_{i 1}, \ldots, x_{i n}\right\}$ the domain associated with the variable $X_{i} . x_{i j}$ denotes the $j$ th instance of $X_{i}$. The universe of discourse is denoted by $\Omega=\times_{X_{i} \in \mathcal{V}} \mathbb{D}_{X_{i}}$, which is the Cartesian product of all variables domain in $\mathcal{V}$. Each element $\omega \in \Omega$ is called an interpretation which represents a possible state of $\Omega$. It is denoted by $\omega=\left(x_{1 i}, \ldots, x_{N j}\right)$. $\phi, \psi \ldots$ represent events, namely subsets of $\Omega$.

\subsubsection{Possibility distribution}

One of basic elements in a possibility theory is the notion of possibility distribution $\pi$ which corresponds to a mapping from $\Omega$ to the scale $[0,1]$. This distribution encodes available knowledge on real world. $\pi(\omega)=1$ means that $\omega$ is completely possible and $\pi(\omega)=0$ means that it is impossible for $\omega$ to represent the real world. A possibilistic scale can be interpreted in ordinal or numerical way. A possibility distribution $\pi$ is said to be $\alpha$-normalized, if its normalization degree, denoted $h(\pi)$ is equal to $\alpha$, namely:

$$
h(\pi)=\max _{\omega} \pi(\omega)=\alpha .
$$

If $\alpha=1$, then $\pi$ is said to be normalized.

Given a possibility distribution $\pi$ on the universe discourse $\Omega$, two dual measures are defined for each event $\phi \subseteq \Omega$ :
- Possibility measure: this measure evaluates to what extent $\phi$ is consistent with our knowledge encoded by $\pi$ :

$$
\Pi(\phi)=\max _{\omega \in \Omega}\{\pi(\omega): \omega \in \phi\}
$$

- Necessity measure: it is the dual of a possibility measure. The necessity measure evaluates at which level $\phi$ is certainly implied by our knowledge represented by $\pi$ :

$$
N(\phi)=1-\Pi(\bar{\phi})
$$

\subsubsection{Possibilistic conditioning}

The possibilistic conditioning consists in the revision of our initial knowledge, encoded by a possibility distribution $\pi$, by the arrival of a new certain information $\phi \subseteq \Omega$. The initial distribution $\pi$ is then replaced by another one, denoted by $\pi^{\prime}=$ $\pi(. \mid \phi)$. The two interpretations of the possibilistic scale (qualitative and quantitative) induce two definitions of possibilistic conditioning [12]: productbased conditioning and min-based conditioning. In this paper, we focus on min-based conditioning defined by [12]:

$\pi\left(\left.\omega\right|_{\min } \phi\right)= \begin{cases}1 & \text { If } \pi(\omega)=\Pi(\phi) \text { and } \omega \in \phi \\ \pi(\omega) & \text { If } \pi(\omega)<\Pi(\phi) \text { and } \omega \in \phi \\ 0 & \text { otherwise }\end{cases}$

We also use a so-called min-based independence relation, as a non-interactivity relation [11]. This relation is obtained by using the min-based conditioning Equation 4 and it is defined by:

$$
\forall x, y, z \Pi(x \wedge y \mid z)=\min (\Pi(x \mid z), \Pi(y \mid z)) .
$$

\subsection{Min-based possibilistic networks}

\subsubsection{Preliminaries}

A min-based possibilistic network [13] over a set of variables $\mathcal{V}$ denoted by $\Pi G_{\text {min }}=(G, \pi)$ is characterized by:

1. A graphical component: which is represented by a Directed Acyclic Graph (DAG) where nodes correspond to variables and arcs represent dependence relations between variables.

2. Numerical components: these components quantify different links in the DAG by using local possibility distributions for each node $X$ in the context of its parents denoted by $\operatorname{Par}(X)$. More precisely:

- For every root node $X(\operatorname{Par}(X)=\emptyset)$, uncertainty is represented by a priori possibility degree $\pi(x)$ for each instance $x \in$ $\mathbb{D}_{X}$, such that $\max _{x} \pi(x)=1$. 
- For the rest of the nodes $(\operatorname{Par}(X) \neq \emptyset)$, uncertainty is represented by the conditional possibility degree $\pi(x \mid \operatorname{par}(X))$ for each instance $x \in \mathbb{D}_{X}$ and $\operatorname{par}(X) \in$ $\mathbb{D}_{\operatorname{Par}(X)}$ (where $\mathbb{D}_{\operatorname{Par}(X)}$ represents Cartesian product of all variables domain in $\operatorname{Par}(X))$, such that $\max _{x} \pi(x \mid \operatorname{par}(X))=$ 1 , for any $\operatorname{par}(X)$.

The set of a priori and conditional possibility degrees induces a unique joint possibility distribution $\pi_{\min }$ defined by:

$$
\pi_{\min }\left(X_{1}, \ldots, X_{N}\right)=\min _{i=1 . . N} \pi\left(X_{i} \mid \operatorname{Par}\left(X_{i}\right)\right) .
$$

The most common task performed on possibilistic networks is possibilistic inference which corresponds to determine how the realization of specific values of some variables affects the remaining variables [14]. The problem of computing posteriori marginal distributions on nodes in arbitrary possibilistic networks is known to be a hard problem [13] except for singly connected graphs which ensure the propagation in polynomial time. In fact, we distinguish two types of these algorithms: one that operates on DAG without loops and others more general which permit the propagation on multiple possibilistic networks. The idea is to transform the initial graph into a junction tree on which the propagation algorithm can be achieved in an efficient way [15]. Other work more recent are based on compilation process [16]. However, other works in probabilistic framework have been proposed $[17,14]$.

\subsubsection{Min-based fusion of possibilistic networks}

Merging uncertain informations [18] is important to exploit complementarities between sources providing thus a global and complete point of view. In this paper, we are interested in conjunctive mode which makes sense if all sources are considered as equally and fully reliable. One of the basic conjunctive operators is the minimum operation ( $\mathrm{min})$. Given two min-based possibilistic networks $\Pi G_{\min }=(G, \pi)$, which induces a joint possibility distribution $\pi_{G}$ and $\Pi G_{\min }^{\prime}=\left(G^{\prime}, \pi^{\prime}\right)$, which induces a joint possibility distribution $\pi_{G^{\prime}}^{\prime}$ then the result of merging $\Pi G_{m i n}$ and $\Pi G_{m i n}^{\prime}$ is the possibilistic network $\Pi G_{\oplus}=\left(G_{\oplus}, \pi_{\oplus}\right)[10]$, which induces a joint possibility distribution $\pi_{G_{\oplus}}$ such that

$$
\forall \omega, \pi_{G_{\oplus}}(\omega)=\min \left(\pi_{G}(\omega), \pi_{G^{\prime}}^{\prime}(\omega)\right)
$$

The syntactic counterpart of the fusion of two possibility distributions, associated with two possibilistic networks, using the min operator is a new minbased possibilistic network definition of which depends on the union of the two initial ones. In [10], the authors propose two main classes for merging min-based possibilistic networks:

- Fusion of same-structure networks: namely where $G=G^{\prime}$, then:
- The resulting network $\Pi G_{\oplus}$ keeps the same structure: $G_{\oplus}=G^{\prime}=G$,

- For each variable $X, \pi_{\oplus}(X \mid \operatorname{Par}(X))=$ $\min \left(\pi(X \mid \operatorname{Par}(X)), \pi^{\prime}(X \mid \operatorname{Par}(X))\right)$.

- Fusion of networks with different structures: in the case where the two networks have different structures, two cases are distinguishable:

- The union of graphs is acyclic: in this case, $\Pi G_{\min }$ and $\Pi G_{\min }^{\prime}$ are first expanded, with additional variables and links, to $\Pi G_{1}=\left(G_{\oplus}=G \cup G^{\prime}, \pi_{1}\right)$ and $\Pi G_{1}^{\prime}=\left(G_{\oplus}=G \cup G^{\prime}, \pi_{1}^{\prime}\right)$ without affecting possibilistiy distribution [10]. Then the fusion of same-structure networks is applied.

- The union of graphs is cyclic: in this case, additional variables are added to eliminate cycles. The new conditional distributions relative to the new variables ensure the equivalence between new and old variables.

For more details on the fusion of possibilistic networks see [10].

\section{Encoding decision problems under uncertainty using possibilistic networks}

A qualitative decision problem [19] is modeled by a finite set of possible states of the world $\mathcal{S}=$ $\left\{s_{1}, \ldots, s_{n}\right\}$, a finite set of consequences $\mathcal{X}$, a set of decisions $D=\left\{d_{1}, \ldots, d_{m}\right\}$, such that each decision $d_{i}: \mathcal{S} \rightarrow \mathcal{X}$ associates for each possible state a consequence. Preferences among consequences are encoded by utility function $\mu: \mathcal{X} \rightarrow \mathcal{U}$ where $\mathcal{U}$ is a preference ordinal scale.

Possibility theory describes uncertain knowledge on different states of the world by means of possibility distributions. It can first represent uncertain knowledge distinguishing what is plausible from what is less plausible. It can also represent preferences over desired or less desired consequences [7]. Therefore, the uncertainty on possible states of the world is expressed by a normalized possibility distribution $\pi$ mapping a set of state variables values into $[0,1]$. Similarly, agent's preferences are represented by means of another possibility distribution $\mu$ (which may not be normalized) mapping a set of consequences into an ordinal scale $\mathcal{U}$, here represented by $[0,1]$. We assume that uncertainty and preferences scales are commensurate [6]. A decision is represented by a function $d$ from $S$ to $X$. The consequence $d(s) \in X$ associated to a decision $d$ on the state $s$ can be evaluated by combining possibility degrees $\pi(s)$ and utilities $\mu(d(s))$ for all possible states of world. Two evaluation criteria have been proposed to achieve such combinations: the pessimistic [20] and the optimistic [21] one. The first one being more cautious than the second one 
for computing optimal decisions.

In [22], the authors have proposed a compact representation of a qualitative decision problems based on possibilistic logic [23]. The logical approach allows to express agent's knowledge and preferences by means of valued logical formula enabling to deduce the corespondent utility and uncertainty functions. Indeed, two weighted propositional logical bases are used: (1) a knowledge base describing what the agent knows and (2) another base describing the consequences satisfying agent's preferences. The proposed logical approach use two types of variables: decision variables and state ones.

Two syntactical approaches have been proposed in [24], based on possibilistic logic, for solving qualitative decision problems: one pessimistic and another optimistic. Both syntactical approaches are respectively in agreement with the semantic definition of the two qualitative possibilistic criteria.

In [25], an algorithm for computing optimal decisions using syntactic possibilistic fusion has been proposed. The proposed approach is based on logical representation of decision problems as in [24]. Our aim in this paper is to propose a new graphical model for representing qualitative decision problems under uncertainty. Our starting point is a possibility distribution $\pi$ and a utility function $\mu$ which represent respectively uncertainty on possible states of the world and agent's preferences. We propose to compactly encoded these two possibility distributions (uncertainty and utility) using two distinct min-based posibilistic networks rather than the two stratified bases: one representing agent's beliefs and the second representing the qualitative utility. The first min-based posibilistic network, denoted by $\Pi K_{\min }=\left(G_{K}, \pi\right)$, represents agent's knowledge and induces a unique possibility distribution $\pi_{K}=\pi$ using Equation 6 . The second min-based possibilistic network, denoted by $\Pi P_{\min }=\left(G_{P}, \mu\right)$, defines agent's preferences and induces a unique qualitative utility $\mu_{P}=\mu$ using also Equation 6 . In the same way as in logical based approach [24], the graphical components $G_{K}$ and $G_{p}$ of the two minbased possibilistic networks $\Pi K_{\min }$ and $\Pi P_{\min }$ are defined on two types of variables: decision variables noted $\mathcal{D}=\left\{D_{1}, \ldots, D_{p}\right\}$ and state variables noted $\mathcal{X}=\left\{X_{1}, \ldots, X_{n}\right\}$. Concerning decision evaluation process, we propose to preserve the two qualitative criteria used in the case of logical representations of qualitative decision problems under uncertainty. Since our new graphical model for representing possibilistic decision making under uncertainty is based on the possibility distribution and qualitative utility then there is no interference on qualitative decision criteria. So, making a decision comes down to choosing a decision $d$ which maximises one of the two qualitative utilities given by [19]:

\section{- Optimistic utility:}

$$
u^{*}(d)=\max _{\omega \in \Omega} \min \left(\pi_{d}(\omega), \mu(\omega)\right)
$$

\section{- Pessimistic utility:}

$$
u_{*}(d)=\min _{\omega \in \Omega} \max \left(n\left(\pi_{d}(\omega)\right), \mu(\omega)\right)
$$

where $n$ represents reversing map on scale $[0,1]$.

In this paper, we only deal with optimistic decision making.

Example 1 Let us consider two min-based possibilistic networks $\Pi K_{\min }$ and $\Pi P_{\min }$.

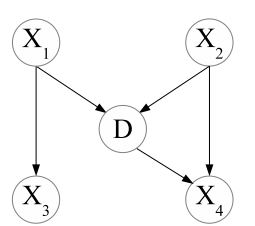

(a)

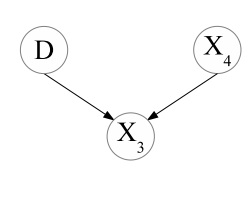

(b)
Figure 1: Example of the acyclic union of networks

1. The agent's knowledge: it is described by the min-based possibilistic network $\Pi K_{\min }=$ $\left(G_{K}, \pi\right)$. Its graphical component $G_{K}$ is given by Figure 1 (a). It contains four possible states of the world $\left\{X_{1}, X_{2}, X_{3}, X_{4}\right\}$ and one decision variable $\{D\}$. The initial possibility distributions associated with $\Pi K_{\min }$ are given by $\mathrm{Ta}$ bles 1, 2, 3 and 4. We suppose that all variables are binary.

\begin{tabular}{|cc||cc|}
\hline$X_{1}$ & $\pi\left(X_{1}\right)$ & $X_{2}$ & $\pi\left(X_{2}\right)$ \\
\hline$x_{1}$ & .5 & $x_{2}$ & .8 \\
$\neg x_{1}$ & 1 & $\neg x_{2}$ & 1 \\
\hline
\end{tabular}

Table 1: Initial possibility distributions $\Pi K_{m i n}$ on $X_{1}$ and $X_{2}$

\begin{tabular}{|ccc|ccc|}
\hline$X_{3}$ & $X_{1}$ & $\pi\left(X_{3} \mid X_{1}\right)$ & $X_{3}$ & $X_{1}$ & $\pi\left(X_{3} \mid X_{1}\right)$ \\
\hline$x_{3}$ & $x_{1}$ & .5 & $\neg x_{3}$ & $x_{1}$ & 1 \\
$x_{3}$ & $\neg x_{1}$ & .6 & $\neg x_{3}$ & $\neg x_{1}$ & 1 \\
\hline
\end{tabular}

Table 2: Initial possibility distributions $\Pi K_{\text {min }}$ on $X_{3} \mid X_{1}$

\begin{tabular}{|cccc|cccc|c|}
\hline$D$ & $X_{1}$ & $X_{2}$ & $\pi\left(D \mid X_{1} X_{2}\right)$ & $D$ & $X_{1}$ & $X_{2}$ & $\pi\left(D \mid X_{1} X_{2}\right)$ \\
\hline$d$ & $x_{1}$ & $x_{2}$ & .1 & $\neg d$ & $x_{1}$ & $x_{2}$ & 1 \\
$d$ & $x_{1}$ & $\neg x_{2}$ & .6 & $\neg d$ & $x_{1}$ & $\neg x_{2}$ & 1 \\
$d$ & $\neg x_{1}$ & $x_{2}$ & .7 & $\neg d$ & $\neg x_{1}$ & $x_{2}$ & 1 \\
$d$ & $\neg x_{1}$ & $\neg x_{2}$ & .4 & $\neg d$ & $\neg x_{1}$ & $\neg x_{2}$ & 1 \\
\hline
\end{tabular}

Table 3: Initial possibility distributions $\Pi K_{\min }$ on $D \mid X_{1} X_{2}$ 


\begin{tabular}{|cccc|cccc|}
\hline$X_{4}$ & $D$ & $X_{2}$ & $\pi\left(X_{4} \mid D X_{2}\right)$ & $X_{4}$ & $D$ & $X_{2}$ & $\pi\left(X_{4} \mid D X_{2}\right)$ \\
\hline$x_{4}$ & $d$ & $x_{2}$ & .1 & $\neg x_{4}$ & $d$ & $x_{2}$ & 1 \\
$x_{4}$ & $d$ & $\neg x_{2}$ & .9 & $\neg x_{4}$ & $d$ & $\neg x_{2}$ & 1 \\
$\neg x_{4}$ & $\neg d$ & $x_{2}$ & .2 & $\neg x_{4}$ & $\neg d$ & $x_{2}$ & .8 \\
$\neg x_{4}$ & $\neg d$ & $\neg x_{2}$ & 1 & $\neg x_{4}$ & $\neg d$ & $\neg x_{2}$ & 1 \\
\hline
\end{tabular}

Table 4: Initial possibility distributions $\Pi K_{\min }$ on $X_{4} \mid D X_{2}$

2. Agent's preferences: are expressed by the minbased possibilistic network $\Pi P_{\min }=\left(G_{P}, \mu\right)$, where its DAG is given by Figure 1 (b). The initial possibility distributions associated with $\Pi P_{\min }$ are given by Tables 5 and 6 .

\begin{tabular}{|cc||cc|}
\hline$D$ & $\mu(D)$ & $X_{4}$ & $\mu\left(X_{4}\right)$ \\
\hline$d$ & .5 & $x_{4}$ & 1 \\
$\neg d$ & 1 & $\neg x_{4}$ & .3 \\
\hline
\end{tabular}

Table 5: Initial possibility distributions $\Pi P_{\min }$ on $D$ and $X_{4}$

\begin{tabular}{|cccc|c|cccc|}
\hline$X_{3}$ & $D$ & $X_{4}$ & $\mu\left(X_{3} \mid D X_{4}\right)$ & $X_{3}$ & $D$ & $X_{4}$ & $\mu\left(X_{3} \mid D X_{4}\right)$ \\
\hline$x_{3}$ & $d$ & $x_{4}$ & 1 & $\neg x_{3}$ & $d$ & $x_{4}$ & .7 \\
$x_{3}$ & $d$ & $\neg x_{4}$ & .6 & $\neg x_{3}$ & $d$ & $\neg x_{4}$ & 1 \\
$\neg x_{3}$ & $\neg d$ & $x_{4}$ & .8 & $\neg x_{3}$ & $\neg d$ & $x_{4}$ & 1 \\
$\neg x_{3}$ & $\neg d$ & $\neg x_{4}$ & .2 & $\neg x_{3}$ & $\neg d$ & $\neg x_{4}$ & 1 \\
\hline
\end{tabular}

Table 6: Initial possibility distributions $\Pi K_{m i n}$ on $X_{3} \mid D X_{4}$

\section{On the computation of optimal optimistic decisions based on min-based fusion}

This section presents the computation of qualitative possibilistic decisions which can be viewed as a data fusion problem of two particular possibility distributions: one representing agent's beliefs and the second representing the qualitative utility. We assume that agent's knowledge and preferences are both represented by two separated min-based possibilistic networks. The first min-based posibilistic network $\Pi K_{\min }=\left(G_{K}, \pi\right)$ represents agent's knowledge. $\Pi K_{\min }$ induces a unique possibility distribution $\pi_{K}$ using Equation 6. The second minbased possibilistic network $\Pi P_{\min }=\left(G_{P}, \mu\right)$ defines agent's preferences. $\Pi P_{\min }$ induces a unique qualitative utility $\mu_{P}$ using Equation 6 .

In what follows, we propose a direct method for computing optimal optimistic decisions based on the fusion of $\pi_{K}$ and $\mu_{P}$ (or $\Pi K_{m i n}$ and $\Pi P_{\text {min }}$ ).

Each set of decision $d$ induces a possibility distribution $\pi_{K_{d}}$ in the following way [6]:

$$
\pi_{K_{d}}(\omega)=\min \left(\pi_{K}(\omega), \pi_{d}(\omega)\right),
$$

where,

$$
\pi_{d}(\omega)= \begin{cases}1 & \text { If } \omega \models d \\ 0 & \text { otherwise }\end{cases}
$$

where $\omega \models d$ means the value of $D$ in $\omega$ is equal to d.

\subsection{Describing optimistic decisions as a fusion process}

We recall that an optimal optimistic decision $d$ is defined by the one that maximizes the expression:

$$
u^{*}(d)=\max _{\omega \in \Omega} \min \left(\pi_{K_{d}}(\omega), \mu_{P}(\omega)\right)
$$

Using equation 8, the optimistic utility decision $u^{*}(d)$ becomes:

$$
u^{*}(d)=\max _{\omega \in \Omega} \min \left(\min \left(\pi_{K}(\omega), \mu_{P}(\omega)\right), \pi_{d}(\omega)\right)
$$

Using technical merging of two min-based possibilistic networks (Equation 7), Equation 11 comes:

$$
u^{*}(d)=\max _{\omega \in \Omega} \min \left(\pi_{G_{\oplus}}(\omega), \pi_{d}(\omega)\right)
$$

Besides, we have recalled in section 3.2.2 how to compute the syntactic counterpart of $\min \left(\pi, \pi^{\prime}\right)$. Then, the syntactic counterpart of $\min \left(\pi_{K}(\omega), \mu_{P}(\omega)\right)$ is the min-based possibilistic network denoted by $\Pi G_{\oplus}=\left(G_{\oplus}, \pi_{\oplus}\right)$ which induces the unique possibility distribution $\pi_{G_{\oplus}}$.

Example 2 The two $D A G_{s}\left(G_{K}\right.$ and $\left.G_{P}\right)$ given in Example 1, Figure 1 have a different structure. Their union is free of cycles, then the result of merging $\Pi K_{\min }$ and $\Pi P_{\min }$ is the min-based possibilistic network $\Pi G_{\oplus}=\left(G_{\oplus}, \pi_{\oplus}\right)$, where $G_{\oplus}$ is given in Figure 2. $G_{\oplus}$ is simply the union of the two graphs of Figure 1.

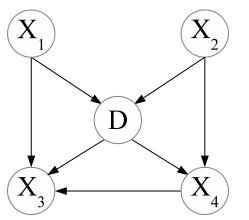

Figure 2: The DAG $G_{\oplus}$

The initial possibility distributions are given by Tables 7, 8, 9 and 10, which are obtained using the minimum of local distributions $\Pi K_{\text {min }}$ and $\Pi P_{\text {min }}$.

\begin{tabular}{|cc||cc|}
\hline$X_{1}$ & $\pi_{\oplus}\left(X_{1}\right)$ & $X_{2}$ & $\pi_{\oplus}\left(X_{2}\right)$ \\
\hline$x_{1}$ & .5 & $x_{2}$ & .8 \\
$\neg x_{1}$ & 1 & $\neg x_{2}$ & 1 \\
\hline
\end{tabular}

Table 7: Initial possibility distributions $\Pi G_{\oplus}$ on $X_{1}$ and $X_{2}$

\begin{tabular}{|llll|llll|}
\hline$D$ & $X_{1}$ & $X_{2}$ & $\pi_{\oplus}\left(D \mid X_{1} X_{2}\right)$ & $D$ & $X_{1}$ & $X_{2}$ & $\pi_{\oplus}\left(D \mid X_{1} X_{2}\right)$ \\
\hline$d$ & $x_{1}$ & $x_{2}$ & .1 & $\neg d$ & $x_{1}$ & $x_{2}$ & 1 \\
$d$ & $x_{1}$ & $\neg x_{2}$ & .1 & $\neg d$ & $x_{1}$ & $\neg x_{2}$ & 1 \\
$d$ & $\neg x_{1}$ & $x_{2}$ & .1 & $\neg d \neg x_{1}$ & $x_{2}$ & 1 \\
$d$ & $\neg x_{1}$ & $\neg x_{2}$ & .1 & $\neg d \neg x_{1}$ & $\neg x_{2}$ & 1 \\
\hline
\end{tabular}

Table 8: Initial possibility distributions $\Pi G_{\oplus}$ on $D$ $X_{1} X_{2}$ 


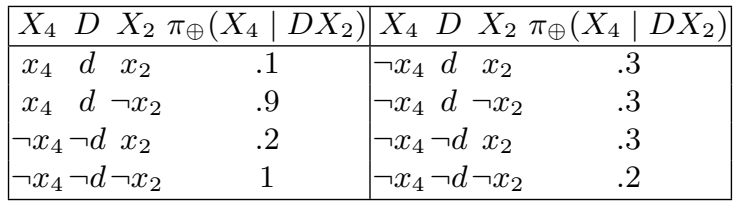

Table 9: Initial possibility distributions $\Pi G_{\oplus}$ on $X_{4} \mid D X_{2}$

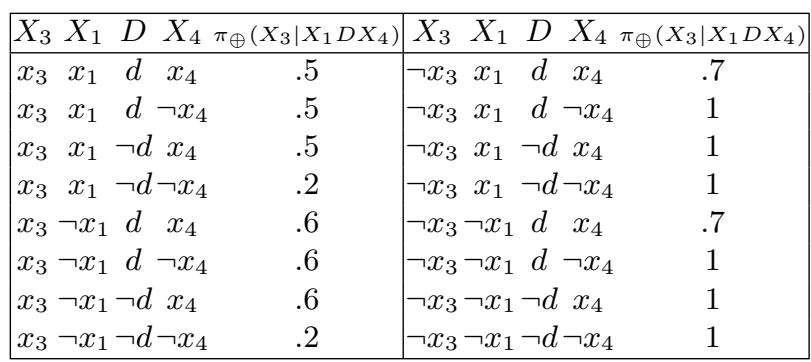

Table 10: Initial possibility distributions $\Pi G_{\oplus}$ on $X_{3} \mid X_{1} D X_{4}$

So, the min-based possibilistic network $\Pi G_{\oplus}$ issued from the fusion phase can be used to generate optimal decisions by maximizing the qualitative utility relative to each decision. This computation is ensured by applying a propagation process based on junction tree.

\subsection{Computing optimal decisions using junction trees}

The computation of $\min \left(\pi_{G_{\oplus}}(\omega), \pi_{d}(\omega)\right)$ is performed using junction tree algorithm on $\Pi G_{\oplus}$. This junction tree is parameterized by some decision $d$. Note that the construction of junction tree is only done once. However, the propagation and the initialization (which are both polynomial) are repeated for each possible decision. The following provides mains steps of our algorithm:

- Building junction tree $\mathcal{J T}$ : min-based propagation algorithms begin by transforming the initial graph $G_{\oplus}$ into a junction tree in three steps [17]:

- Moralization of the initial graph $G_{\oplus}$ : consists of the creation of an undirected graph from the initial one by adding links between the parents of each variable.

- Triangulation of the moral graph: allows to identify sets of variables that can be grouped as clusters noted $C_{i}$. Several heuristics have been proposed in order to find the best triangular graph which minimizes the size of clusters.

- Construction of the optimal junction tree $\mathcal{J T}$ : the junction tree is built by connecting the clusters identified in the previous step such that all clusters on the path between any two clusters $C_{i}$ and $C_{j}$ should contain $C_{i} \cap C_{j}$. Once adjacent clusters have been identified, between each pair of clusters $C_{i}$ and $C_{j}$, a separator $S_{i j}$ containing their common variables, will be inserted.

This step (building junction tree) is the same as in standard possibilistic or probabilistic networks. However, as we will see the initialization step is not the same.

- Initialization for a given decision $d$ : once the junction tree is built, we proceed to its quantification taking into account the decision $d$ as follows:

- For each cluster $C_{i}$ (resp. $S_{i j}$ ), $\pi_{C_{i}}^{I} \leftarrow 1$ (resp. $\pi_{S_{i j}}^{I} \leftarrow 1$ ),

- for each variable $X_{i}$, select a cluster $C_{i}$ containing $\left\{X_{i}\right\} \cup \operatorname{Par}\left(X_{i}\right), \pi_{C_{i}}^{I} \leftarrow$ $\min \left(\pi_{C_{i}}^{I}, \pi_{\oplus}\left(X_{i} \mid \operatorname{Par}\left(X_{i}\right)\right)\right)$

- encode the evidence $D=\{d\}$ as likelihood $\Lambda_{D}(d)$ :

$$
\Lambda_{D}(d)= \begin{cases}1 & \text { If } D \text { is instanciated as } d \\ 0 & \text { If } D \text { is instanciated by a } \\ & \text { value diffrent from } d\end{cases}
$$

- identify a cluster $C_{i}$ containing $D: \pi_{C_{i}}^{I} \leftarrow$ $\min \left(\pi_{C_{i}}^{I}, \Lambda_{D}\right)$

Note that Equation 13 does not appear in standard initialization of junction trees associated with standard min-based possibilistic networks. It is proper to our framework. By entering the fact $D=\{d\}$, the junction tree $\mathcal{J T}$ encodes $\pi_{\mathcal{J T}}(\omega)=\min \left(\pi_{G_{\oplus}}(\omega), \pi_{d}(\omega)\right)$. Then the qualitative utility associated to a decision $d$ is summarized by the following proposition:

Proposition 1 Let $\Pi K_{\min }=\left(G_{K}, \pi\right)$ be a min-based possibilistic network representing agent's beliefs and $\Pi P_{\text {min }}=\left(G_{P}, \mu\right)$ be a minbased possibilistic network representing agent's preferences. Let $\Pi G_{\oplus}=\left(G_{\oplus}, \pi_{\oplus}\right)$ be the result of merging $\Pi K_{\min }$ and $\Pi P_{\min }$ using the min operator. Let $\mathcal{J} \mathcal{T}$ be the junction tree corresponding to $\Pi G_{\oplus}$ generated using the above initialization procedure. Then,

$$
u^{*}(d)=\max _{\omega \in \Omega} \pi_{\mathcal{J}} \mathcal{T}(\omega) .
$$

where $u^{*}(d)$ is given in Equation 12.

Hence, after the initialization step, the junction tree really encodes the possibilistic optimistic decision. The next step is used to apply propagation algorithm in order to efficiently determine the value of $u^{*}(d)$.

- Global propagation: after the initialization step of $\mathcal{J} \mathcal{T}$, the global propagation is performed in order to make it globally consistent, namely:

$$
\max _{C_{i} \backslash S_{i j}} \pi_{C_{i}}^{t}=\pi_{S_{i j}}^{t}=\max _{C_{j} \backslash S_{i j}} \pi_{C_{j}}^{t} .
$$


The global propagation is ensured via a message passing mechanism between clusters which is based on two main phases: collection and distribution of the evidence [26]. Once stability is reached, the computation of qualitative utility relative to a decision $d$ can be achieved as follows:

Proposition 2 Let $\Pi K_{\min }=\left(G_{K}, \pi\right)$ be a min-based possibilistic network representing agent's beliefs and $\Pi P_{\text {min }}=\left(G_{P}, \mu\right)$ be a minbased possibilistic network representing agent's preferences. Let $\Pi G_{\oplus}=\left(G_{\oplus}, \pi_{\oplus}\right)$ be the result of merging $\Pi K_{\min }$ and $\Pi P_{\min }$ using the min operator. Let $\mathcal{J} \mathcal{T}$ be the junction tree corresponding to $\Pi G_{\oplus}$ generated using the above global propagation procedure. Then, the computation of optimistic decisions amounts to compute a normalization degree of $\mathcal{J} \mathcal{T}$ :

$$
u^{*}(d)=\max _{C_{i}} \pi_{C_{i}} .
$$

The optimal optimistic decisions are those maximizing the qualitative utility. The computation of these optimal optimistic decisions is obtained using the following algorithm.

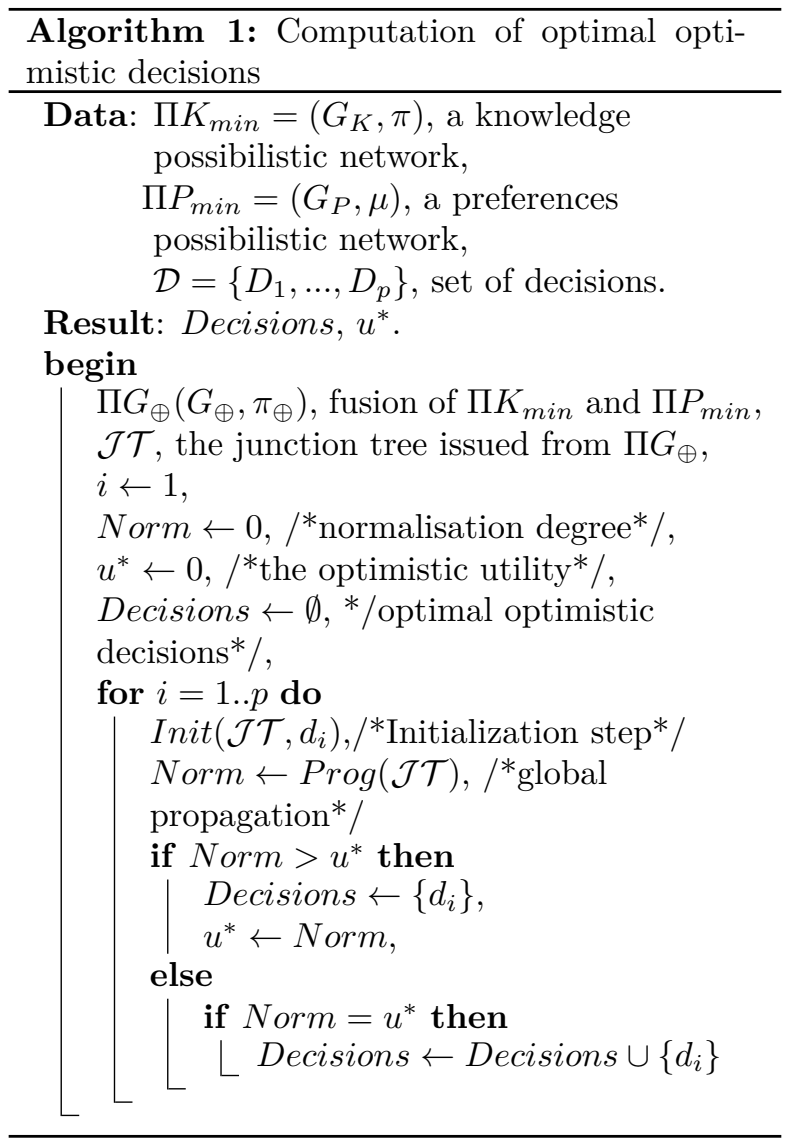

In algorithm 1 , the function $\operatorname{Init}(\mathcal{J T}, \boldsymbol{d})$ corresponds to the initialization step. It has two parameters: the junction tree $\mathcal{J} \mathcal{T}$ issued from $\Pi G_{\oplus}$ and a decision $d$ which will parameterize $\mathcal{J T}$.

Similarly, the function $\operatorname{Prog}(\boldsymbol{J T})$ corresponds to the global propagation and returns normalization degree relative to $\mathcal{J} \mathcal{T}$.
Example 3 Let us continue Example 2. We need to compute the optimal optimistic decision $D=\{d, \neg d\}$. We first begin by constructing the junction tree (see Figure 3) associated with the graph $G_{\oplus}$ (Figure 2) representing the fusion of $\Pi K_{\min }$ and $\Pi P_{\min }$. The resulting junction tree contains two clusters $C_{1}=\left\{X_{1}, X_{2}, X_{4}, D\right\}$ and $C_{2}=\left\{X_{1}, X_{3}, X_{4}, D\right\}$ and their separator $S_{12}=$ $\left\{X_{1}, X_{4}, D\right\}$.

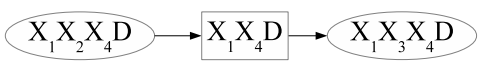

Figure 3: The junction tree associated with $G_{\oplus}$ of example 2

For each decision value $D=\{d, \neg d\}$, we need to run the propagation algorithm in order to compute the normalization degree associated with the junction tree. From the initialization procedure, we get: $\pi_{C_{1}}=\min \left(1, \pi_{\oplus}\left(X_{1}\right), \pi_{\oplus}\left(X_{2}\right), \pi_{\oplus}\left(X_{4} \mid D X_{2}\right)\right)$, $\pi_{C_{2}}=\min \left(1, \pi_{\oplus}\left(X_{3} \mid D X_{1} X_{4}\right), \Lambda_{D}\right)$.

Step $1(\boldsymbol{D}=\{\boldsymbol{d}\})$ : In this case, the fact $D=d$ is encoded as likelihood as follows:

$$
\Lambda_{D}(d)= \begin{cases}1 & \text { If } D \text { is instanciated as } d \\ 0 & \text { If } D \text { is instanciated as } \neg d\end{cases}
$$

Once the junction tree is quantified, then the global propagation allows to compute the normalization degree of the junction tree which corresponds to the normalization degree of any cluster. Using this procedure, we obtain:

$$
u^{*}(d)=\max _{C_{1}} \pi_{C_{1}}=\max _{C_{2}} \pi_{C_{2}}=.7
$$

Step $2(\boldsymbol{D}=\{\neg \boldsymbol{d}\})$ : We repeat the same procedure described in the previous step, with:

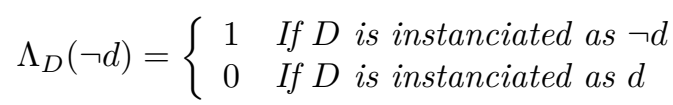

then, we get:

$$
u^{*}(\neg d)=\max _{C_{1}} \pi_{C_{1}}=\max _{C_{2}} \pi_{C_{2}}=1
$$

Thus, we can conclude that the optimal decision $D^{*}=\neg d$ with the maximal qualitative utility equal to 1

\section{Conclusion}

In this paper, we have first proposed a new graphical model for representing possibilistic decision making under uncertainty in compact way using possibilistic networks. This new graphical model benefits from existing tools for possibilistic networks. Secondly, we have proposed a new approach for computing optimal optimistic decisions in the new possibilistic graphical framework. Our approach first merges 
possibilistic networks associated with available uncertain knowledge and possibilistic networks associated with agent's preferences. We then showed that computing optimistic decisions comes down to compute a normalization degree of the junction tree associated to the result graph of merging agent's beliefs and preferences networks. This allows an efficient computation of optimal decisions. As a future work, we plan to apply our solution to deal with the pessimistic decisions for possibilistic decision problems.

\section{References}

[1] P.P. Shenoy. A comparison of graphical techniques for decision analysis. European Journal of Operational Research, 78:1-21, 1994.

[2] R. A Howard and J. E Matheson. Influence Diagrams. In Readings on the Principles and Applications of Decision Analysis, pages 721762. Strategic Decisions Group, 1984.

[3] J.A. Tatman and R.D. Shachter. Dynamic programming and influence diagrams. IEEE Transactions on Systems, Man and Cybernetics, 20(2):365-379, Mars 1990.

[4] N.L. Zhang. Probabilistic inference in influence diagrams. In Computational Intelligence, pages 514-522, 1998.

[5] D. Dubois and H. Prade. Possibility Theory: An Approach to Computerized Processing of Uncertainty . Plenum Press, New York, 1988.

[6] D. Dubois and H. Prade. Possibility theory as a basis for qualitative decision theory. In Proceedings of the 14 th international joint conference on Artificial intelligence - Volume 2, pages 1924-1930, San Francisco, CA, USA, 1995. Morgan Kaufmann Publishers Inc.

[7] L. Garcia and R. Sabbadin. Diagrammes d'influence possibilistes. Revue d'Intelligence Artificielle, 21(4):521-554, 2007.

[8] P.H. Giang and P.P. Shenoy. Two axiomatic approaches to decision making using possibility theory. European Journal of Operational Research, 162(2):450-467, 2005.

[9] W. Guezguez, N. Ben Amor, and K. Mellouli. Qualitative possibilistic influence diagrams based on qualitative possibilistic utilities. European Journal of Operational Research, 195(1):223-238, May 2009.

[10] S. Benferhat and F. Titouna. Min-based fusion of possibilistic networks. In Fourth Conference of the European Society for Fuzzy Logic and Technology(EUSFLAT'05), pages 553-558, 2005.

[11] L. Zadeh. Fuzzy sets as a basis for a theory of possibility. Fuzzy Sets and Systems, 1:3-28, 1978.

[12] B. Bouchon-Meunier, G. Coletti, and C. Marsala. Independence and possibilis- tic conditioning. Annals of Mathematics and Artificial Intelligence, 35:107-123, May 2002.

[13] C. Borgelt, J. Gebhardt, and R. Kruse. Inference methods. In Handbook of Fuzzy Computation, chapter F1.2. Institute of Physics Publishing, Bristol, United Kingdom, 1998.

[14] C. Huang and A. Darwiche. Inference in belief networks: A procedural guide. Int. J. Approx. Reasoning, 15(3):225-263, 1996.

[15] N. Ben Amor, S. Benferhat, and K. Mellouli. Anytime propagation algorithm for min-based possibilistic graphs. Soft Comput., 8(2):150 161, 2003.

[16] R. Ayachi, N. Ben Amor, S. Benferhat, and R. Haenni. Compiling possibilistic networks: Alternative approaches to possibilistic inference. CoRR, abs/1203.3465, 2012.

[17] A. Darwiche. Modeling and Reasoning with Bayesian Networks. Cambridge University Press, New York, NY, USA, 1st edition, 2009.

[18] D. Dubois and H. Prade. Possibility theory and data fusion in poorly informed environments. Control Engineering Practice, 2(5):811823, 1994.

[19] D. Dubois, L. Godo, H. Prade, and A. Zapico. Making decision in a qualitative setting: From decision under uncertainty to case-based decision, 1998.

[20] T. Whalen. Decision making under uncertainty with various assumptions about available information. IEEE Trans. on systems, Man and Cybernetics, (14):888-900, 1984.

[21] R. R Yager. Possibilistic decision making. IEEE Trans. on systems, Man and Cybernetics, (09):388-392, 1979.

[22] D. Dubois, D. Le Berre, H. Prade, and R. Sabbadin. Logical representation and computation of optimal decisions in a qualitative setting. In AAAI-98, pages 588-593, 1998.

[23] D. Dubois, J. Lang, and H. Prade. Possibilistic logic. In Handbook of Logic in Artificial Intelligence and Logic Programming, (D. Gabbay et al., eds, 3, Oxford University Press:pages 439513, 1994.

[24] R. Sabbadin. Une approche logique de la résolution de problèmes de décision sous incertitude basée sur les atms. 11ème congrès Reconnaissance des Formes et Intelligence Artificielle (RFIA'98), pages 391-400, 20-22 janvier 1998.

[25] S. Benferhat, F. Haned-Khellaf, A. Mokhtari, and I. Zeddigha. Using syntactic possibilistic fusion for modeling optimal optimistic qualitative decision. In IFSA/EUSFLAT Conf., pages 1712-1716, jul 2009.

[26] N. Ben Amor. Qualitative possibilistic graphical models : from independence to propagation algorithms. Thèse de doctorat, ISG - Université de Tunis, Tunis, juin 2002. 